\title{
Circulating Tumor Cell Number Is Associated with Primary Tumor Volume in Patients with Lung Adenocarcinoma
}

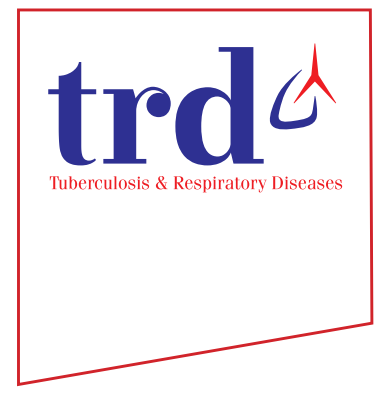

\author{
Byung Ju Kang, M.D., Ph.D. ${ }^{1}{ }^{\mathbb{D}}$, Seung Won Ra, M.D., Ph.D. ${ }^{1}$, Kyusang Lee, Ph.D. ${ }^{2}$, Soyeoun Lim, \\ M.D., M.S. ${ }^{3}$, So Hee Son, M.S. ${ }^{2}$, Jong-Joon Ahn, M.D., Ph.D. ${ }^{1}{ }^{\mathbb{D}}$ and Byung Chul Kim, Ph.D. ${ }^{2}$ \\ ${ }^{1}$ Department of Internal Medicine, Ulsan University Hospital, University of Ulsan College of Medicine, Ulsan, ${ }^{2}$ Liquid Biopsy \\ and Precision Medicine Division, Clinomics Inc., Ulsan, ${ }^{3}$ Department of Radiology, Ulsan University Hospital, Ulsan, Korea
}

Background: Circulating tumor cells (CTCs) are frequently detected in patients with advanced-stage malignant tumors and could act as a predictor of poor prognosis. However, there is a paucity of data on the relationship between CTC number and primary tumor volume in patients with lung cancer. Therefore, our study aimed to evaluate the relationship between CTC number and primary tumor volume in patients with lung adenocarcinoma.

Methods: We collected blood samples from 21 patients with treatment-naive lung adenocarcinoma and 73 healthy individuals. To count CTCs, we used a CTC enrichment method based on fluid-assisted separation technology. We compared CTC numbers between lung adenocarcinoma patients and healthy individuals using propensity score matching, and performed linear regression analysis to analyze the relationship between CTC number and primary tumor volume in lung adenocarcinoma patients.

Results: CTC positivity was significantly more common in lung adenocarcinoma patients than in healthy individuals $(\mathrm{p}<0.001)$. The median primary tumor volume in CTC-negative and CTC-positive patients was $10.0 \mathrm{~cm}^{3}$ and $64.8 \mathrm{~cm}^{3}$, respectively. Multiple linear regression analysis showed that the number of CTCs correlated with primary tumor volume in lung adenocarcinoma patients $(\beta=0.903, p=0.002)$. Further subgroup analysis showed a correlation between CTC number and primary tumor volume in patients with distant $(\mathrm{p}=0.024)$ and extra-thoracic $(\mathrm{p}=0.033)$ metastasis (not in patients with distant metastasis).

Conclusion: Our study showed that CTC numbers may be associated with primary tumor volume in lung adenocarcinomas patients, especially in those with distant metastasis.

Keywords: Neoplastic Cells, Circulating; Tumor Burden; Adenocarcinoma of Lung

Address for correspondence: Jong-Joon Ahn, M.D., Ph.D.

Department of Internal Medicine, Ulsan University Hospital, University of Ulsan College of Medicine, 877 Bangeojinsunhwando-ro, Dong-gu, Ulsan 44033, Korea

Phone: 82-52-250-8870, Fax: 82-52-250-7048, E-mail: drahnjj@gmail.com

Address for correspondence: Byung Chul Kim, Ph.D.

Liquid Biopsy and Precision Medicine Division, Clinomics Inc., 50 UNIST-gil, Ulsan 44919, Korea

Phone: 82-70-8286-6966, Fax: 82-31-8066-7274, E-mail: bckim@clinomics.co.kr

Received: Jul. 6, 2019, Revised: Jul. 30, 2019, Accepted: Sep. 16, 2019

(a) It is identical to the Creative Commons Attribution Non-Commercial License (http://creativecommons.org/licenses/by-nc/4.0/). 


\section{Introduction}

Lung cancer is the leading cause of cancer-related death worldwide, and non-small cell lung cancer (NSCLC) contributes to $85 \%$ of these deaths ${ }^{1,2}$. In recent years, the incidence of lung adenocarcinoma has markedly increased among NSCLC patients ${ }^{3}$. Although many genetic mutations and translocations that can be used for targeted therapies for lung adenocarcinoma have been identified, the 5 -year survival rate remains low, and many patients present with advanced-stage disease at initial diagnosis .

Circulating tumor cells (CTCs) are tumor cells that have been released into the peripheral blood from either a primary lesion or a metastatic site. These cells are involved in metastatic spread, which leads to the dissemination of the disease to secondary sites ${ }^{5}$. Detection and monitoring of CTCs could be a valid and non-invasive method for early detection of tumors, prognosis prediction, and assessment of treatment options in patients with lung cancer ${ }^{6,7}$.

Several studies have examined CTCs in NSCLC patients. These studies found that CTC numbers are higher at advanced tumor stages ${ }^{8-10}$, higher CTC numbers correlate with a poor prognosis for survival ${ }^{11}$, and CTC number is positively correlated with the number of metastasis sites ${ }^{2}$. However, few studies have examined the relationship between CTC number and primary tumor volume. The aim of our study was to evaluate the relationship between CTC number and primary tumor volume in patients with lung adenocarcinoma.

\section{Materials and Methods}

\section{Study design, participants, and clinical parameters}

We performed CTC analysis on a cohort of patients with histologically confirmed, treatment-naive lung adenocarcinoma and healthy individuals, and conducted a retrospective review. We enrolled lung adenocarcinoma patients from Ulsan University Hospital (Ulsan, Korea), diagnosed between October 2015 and June 2017, and healthy outpatients from the "Genome Korea In Ulsan" during the same period. Among the healthy individuals, we excluded patients with a prior or current history of malignancy on chest radiography, chest computed tomography, abdominal ultrasonography, esophagogastroduodenoscopy, and colonoscopy, and individuals under 50 years of age. One pulmonologist reviewed all the patients' clinical data, including age, sex, clinical stage, TNM sub-stage, metastasis sites, radiographic findings, histologic findings, and overall survival. This review was performed more than twice. Clinical stage was determined according to the 8th edition of the TNM classification for lung cancer. Primary tumor volume measurements had been performed by an experienced chest radiologist using a high-performance workstation (TeraRecon Aquarius iNtuition, TeraRecon, Foster City, CA, USA) (Figure 1). This study was approved by the Institutional Review Board at Ulsan University Hospital (IRB No. 2018-04-028). All patients provided written informed consent before blood sampling.

\section{CTC enrichment and enumeration}

The CellSearch system (Menarini Silicon Biosystems, Bologna, Italy) remains the only Food and Drug Administration-

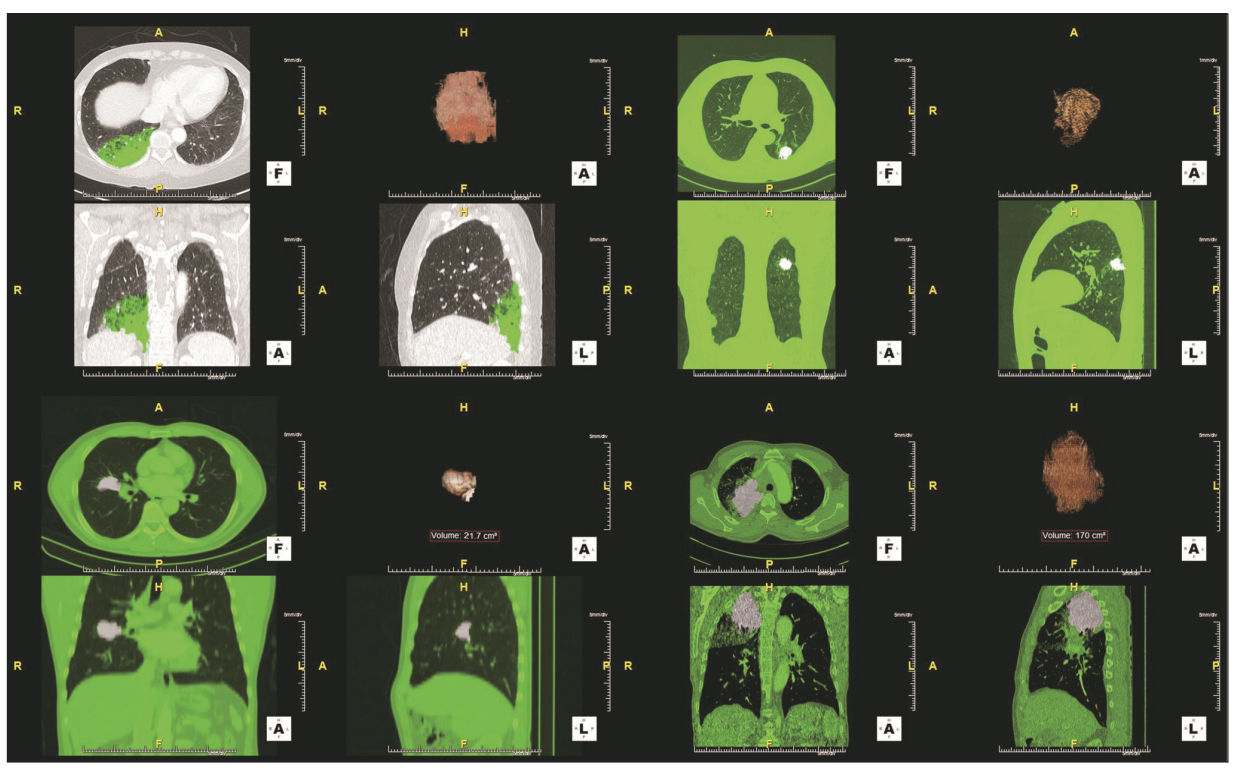

Figure 1. Four cases of primary tumor volume measurement using a high-performance workstation. The transverse (left upper, F), coronal (left lower, A), and sagittal (right lower, L) area of the tumor was examined using computed tomography attenuation values. Then, the device automatically reconstructed 3D images of the tumor (right upper, A). 
approved CTC isolation system proven to have both assay and clinical/biological validity in cancer. However, owing to the low expression of epithelial cell adhesion molecule (EpCAM), there are limitations to the use of the CellSearch system in detecting CTCs in cases of NSCLC. Therefore, no standard method exists for detecting CTCs in cases of NSCLC in clinical practice. We performed CTC enrichment and enumeration using the CD-PRIME platform (Clinomics Inc., Ulsan, Korea), which is based on a fluid-assisted separation technology (FAST)-based CTC enrichment method ${ }^{12}$. Peripheral blood samples were obtained from lung adenocarcinoma and healthy patients ( $3 \mathrm{~mL}$ and $6 \mathrm{~mL}$, respectively). Samples were collected in ethylenediaminetetraacetic acid-coated vacutainer tubes (BD Biosciences, Franklin Lakes, NJ, USA). CTC enrichment was performed within 2 hours after blood sampling. The separation of peripheral blood mononuclear cells (PBMCs) from peripheral blood was accomplished by density gradient centrifugation at $800 \times \mathrm{g}$ for 15 minutes at room temperature using a standard Ficoll-Paque Plus protocol (GE Healthcare, Uppsala, Sweden). Each PBMC sample was diluted with an equal volume of phosphate-buffered saline and was then applied to a CD-CTC Solo disc cartridge of the CD-PRIME system (Clinomics Inc.). CTCs were captured on a membrane within the CD-CTC Solo disc cartridge. To count the captured CTCs, immunofluorescence staining was performed on the disc. CTCs were identified using the following commonly accepted criteria: visible 4,6-diamidino2-phenylindole nuclear staining, positive staining of cytokeratin or EpCAM in the cytoplasm, and negative staining of CD45 (Figure 2A). Isolated CTCs were characterized and enumerated using an automated fluorescence microscope scanning system, BioView Duet workstation (BioView Ltd., Rehovot, Israel) (Figure 2B). Although the CellSearch system defined "CTC positive" as $\geq 2$ CTCs in $7.5 \mathrm{~mL}$ blood, we defined a patient to be "CTC positive" when the total number of CTCs was $\geq 1$ CTCs in $3 \mathrm{~mL}$ of the subject's blood sample based on previous FAST-based CTC analysis results ${ }^{11,12}$.

\section{Statistical analysis}

Continuous variables were expressed as median (range) and compared using the Mann-Whitney U test. Categorical variables were expressed as numbers (\%) and compared using the chi-square test. We conducted linear regression analysis to examine factors that could be related to CTC number in patients with lung adenocarcinoma. Sex, age, and possible influence factors for CTC number screened out $(\mathrm{p}<0.1$ in simple linear regression) were subjected to multiple linear regression analysis, and we also checked the variance inflation factors, which were used to detect multicollinearity. To perform subgroup analysis, we classified lung adenocarcinoma patients according to the extent of distant metastasis, as follows: without distant metastasis, with distant metastasis, and with extrathoracic metastasis. Then, we analyzed the relationships between clinical factors and CTC number using scatter diagrams and simple linear regression analysis. Additionally, we performed analysis of covariance (ANCOVA) to check for the interaction between clinical factors and the extent of distant metastasis.

To establish the clinical utility of CTC enumeration using the FAST method in lung adenocarcinoma patients, we compared CTC results from both lung adenocarcinoma patients and healthy individuals. Since volumes of samples from healthy patients were double that of samples from lung adenocarcinoma patients, we corrected the CTC number obtained from healthy individuals (total CTC divided by 2 ). To reduce potential confounding effects, we adjusted for differences in sex and age between groups using propensity match$\mathrm{ing}^{13}$. We developed propensity score-matched pairs using the Greedy 5/1-digit match algorithm based on propensity scores
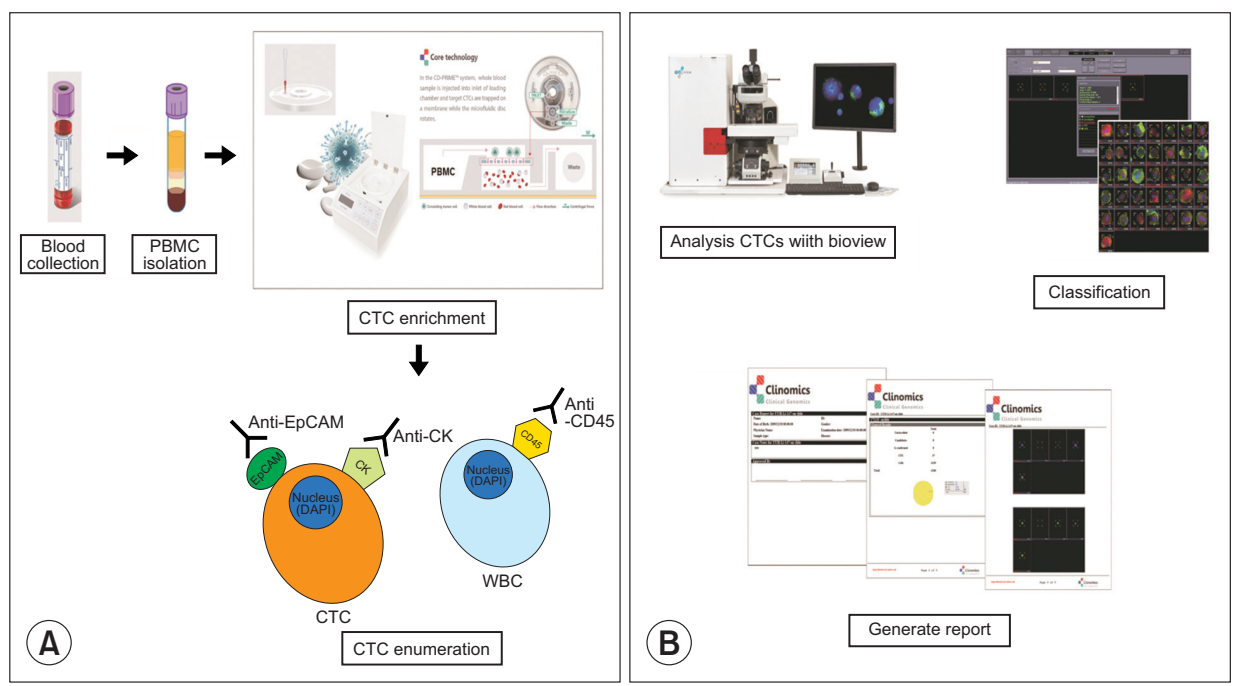

Figure 2. Workflow of circulating tumor cell (CTC) enrichment and enumeration (A), and CTC analysis (B) by the BioView Duet Workstation. WBC: white blood cell; PBMC: peripheral blood mononuclear cell. 
without replacement (a 1:1 match) ${ }^{14}$. A p-value of $<0.05$ was considered statistically significant. All statistical analyses were conducted using SPSS Statistics for Windows version 24.0 (IBM Corp., Armonk, NY, USA).

\section{Results}

Initially, for this study, 49 treatment-naive lung adenocarcinoma patients and 140 healthy individuals were enrolled. We excluded lung adenocarcinoma patients for whom CTCs were analyzed using other methods ( $\mathrm{n}=28)$. Additionally, healthy individuals with current or previous malignancy histories $(\mathrm{n}=16)$ and individuals younger than 50 years old $(\mathrm{n}=51)$ were excluded. Finally, 21 lung adenocarcinoma patients and 73 healthy individuals were examined (Figure 3).

\section{Characteristics of lung adenocarcinoma patients}

The baseline characteristics of lung adenocarcinoma patients are shown in Table 1. The most common clinical stage of lung adenocarcinoma patients was IV, followed by I. Among the 21 lung adenocarcinoma patients, nine (42.9\%) were CTCpositive. The median primary tumor volume was $10.0 \mathrm{~cm}^{3}$ for CTC-negative patients and $64.8 \mathrm{~cm}^{3}$ for CTC-positive patients $(\mathrm{p}=0.102)$. CTC-positive patients had more advanced $\mathrm{T}\left(\mathrm{T}_{1}\right.$ $2 / 9, T_{2} 0 / 9, T_{3} 1 / 9$, and $T_{4} 6 / 9$ vs. $T_{1} 5 / 12, T_{2} 4 / 12, T_{3} 1 / 12$, and $\left.\mathrm{T}_{4} 2 / 12 ; \mathrm{p}=0.036\right)$ and $\mathrm{M}$ stages $\left(\mathrm{M}_{0} 2 / 9, \mathrm{M}_{1 \mathrm{a}} 4 / 9, \mathrm{M}_{1 \mathrm{~b}} 1 / 9\right.$, and $\mathrm{M}_{\mathrm{lc}} 2 / 9$ vs. $\mathrm{M}_{0} 9 / 12, \mathrm{M}_{\mathrm{la}} 0 / 12, \mathrm{M}_{\mathrm{lb}} 0 / 12$, and $\mathrm{M}_{\mathrm{lc}} 3 / 12 ; \mathrm{p}=0.009$ ) than CTC-negative patients. However, there were no significant differences between CTC-negative and CTC-positive patients in terms of age, sex, smoking status, clinical stage, $\mathrm{N}$ category, and metastasis sites.

CTC positivity was more common (stage IV [7/10] vs. stage I-III [2/11]: odds ratio, 10.500 [95\% confidence interval, 1.360-81.053]; $\mathrm{p}=0.030$ ) and CTC counts were higher (stage IV 1 [range, $0-12$ ] vs. stage I-III 0 [range, $0-3$ ]; $p=0.036$ ) in lung adenocarcinoma patients with distant metastasis than in those without distant metastasis (Table 1).

\section{CTCs in lung adenocarcinoma patients and healthy individuals}

The characteristics of lung adenocarcinoma patients and healthy individuals before and after propensity matching are shown in Table 2. CTCs were only captured in lung adenocarcinoma patients after propensity score matching. When we assessed the diagnostic performance of CTC number by constructing a receiver operating characteristic (ROC) curve, the area under ROC curves (AUC) for the differentiation between lung adenocarcinoma patients and healthy individuals was found to be 0.714 ( $\mathrm{p}=0.017$ ). The sensitivity, specificity, and accuracy for the diagnosis of lung adenocarcinoma were $42.9 \%$, $91.8 \%$, and $80.9 \%$ respectively (Figure 4 ).

\section{Relationship of CTC number and primary tumor volume}

When we performed linear regression analysis to analyze the relationship of CTC numbers with clinical markers, CTC number was found to be positively correlated with primary tumor volume in simple $(\beta=0.736, p<0.001)$ and multiple

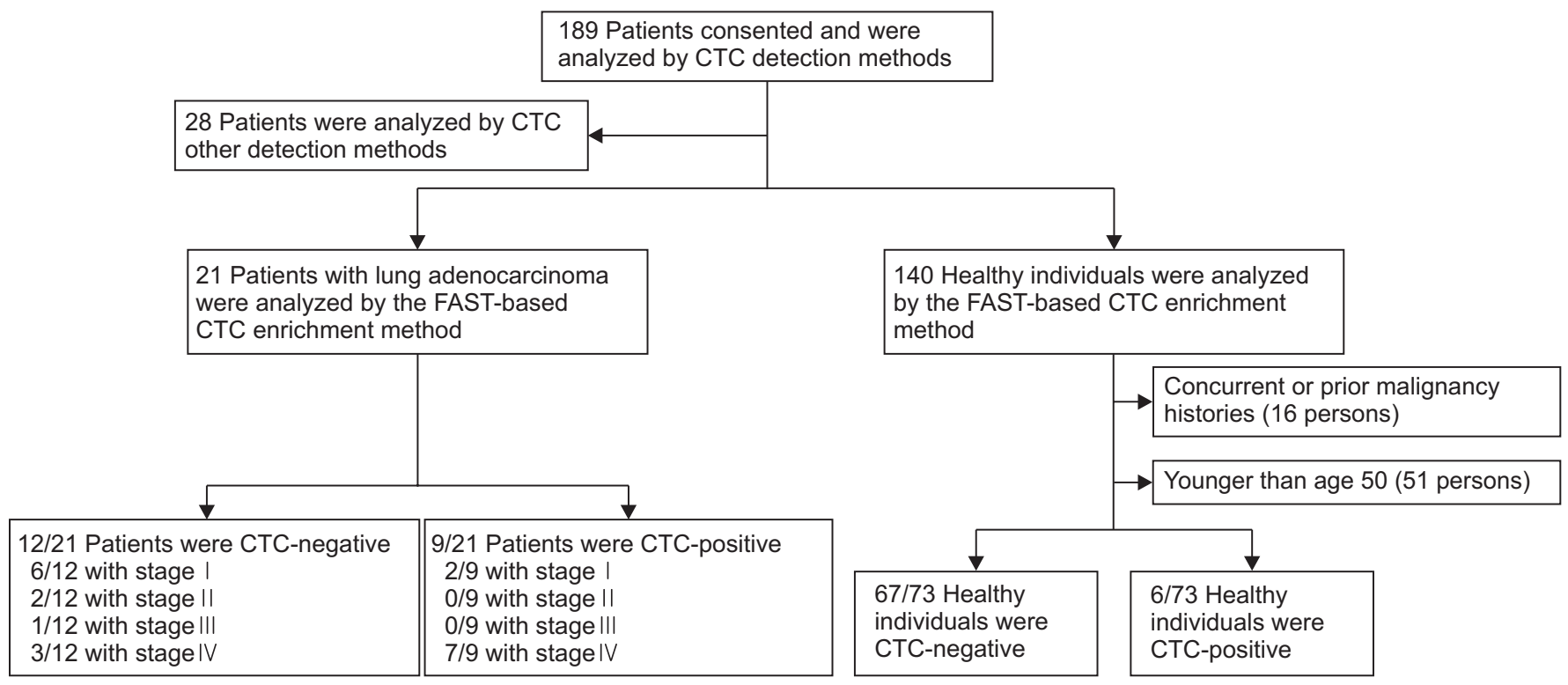

Figure 3. Schematic flow chart of study population selection. CTC: circulating tumor cell; FAST: fluid-assisted separation technology. 
Table 1. Baseline characteristics of lung adenocarcinoma patients with CTC-negative and CTC-positive

\begin{tabular}{|c|c|c|c|c|}
\hline Characteristic & $\begin{array}{l}\text { Total } \\
(n=21)\end{array}$ & $\begin{array}{l}\text { CTC-negative patients } \\
\qquad(\mathrm{n}=12)\end{array}$ & $\begin{array}{l}\text { CTC-positive patients } \\
\qquad(\mathrm{n}=9)\end{array}$ & p-value* \\
\hline Age, yr & $65(54-85)$ & $68(59-72)$ & $62(61-69)$ & 0.722 \\
\hline Male sex & $12(57.1)$ & $7(58.3)$ & $5(55.6)$ & 0.999 \\
\hline Smoking status & & & & 0.999 \\
\hline Non-smoker & $9(42.9)$ & $5(41.7)$ & $4(44.4)$ & \\
\hline Ex- and current smoker & $12(57.1)$ & $7(58.3)$ & $5(55.6)$ & \\
\hline Primary tumor volume, $\mathrm{cm}^{3}$ & $13.1(3.2-324.0)$ & $10.0(3.7-48.1)$ & $64.8(6.7-172.5)$ & 0.102 \\
\hline Clinical stage & & & & 0.058 \\
\hline I & $8(38.1)$ & $6(50.0)$ & $2(22.2)$ & \\
\hline II & $2(9.5)$ & $2(16.7)$ & $0(0.0)$ & \\
\hline III & $1(4.8)$ & $1(8.3)$ & $0(0.0)$ & \\
\hline IV & $10(47.6)$ & $3(25.0)$ & $7(77.8)$ & \\
\hline Primary tumor & & & & 0.036 \\
\hline $\mathrm{T} 1$ & $7(33.3)$ & $5(41.7)$ & $2(22.2)$ & \\
\hline $\mathrm{T} 2$ & $4(19.0)$ & $4(33.3)$ & $0(0.0)$ & \\
\hline T3 & $2(9.5)$ & $1(8.3)$ & $1(11.1)$ & \\
\hline $\mathrm{T} 4$ & $8(38.1)$ & $2(16.7)$ & $6(66.7)$ & \\
\hline Lymph node involvement & & & & 0.547 \\
\hline No & $11(52.4)$ & $7(58.3)$ & $4(44.4)$ & \\
\hline N1 & $1(4.8)$ & $1(8.3)$ & $0(0.0)$ & \\
\hline $\mathrm{N} 2$ & $3(14.3)$ & $1(8.3)$ & $2(22.2)$ & \\
\hline N3 & $6(28.6)$ & $3(25.0)$ & $3(33.3)$ & \\
\hline Distant metastasis & & & & 0.009 \\
\hline M0 & $11(52.4)$ & $9(95.0)$ & $2(22.2)$ & \\
\hline Mla & $4(19.0)$ & $0(0)$ & $4(44.4)$ & \\
\hline Mlb & $1(4.8)$ & $0(0)$ & $1(11.1)$ & \\
\hline Mlc & $5(23.8)$ & $3(25.0)$ & $2(22.2)$ & \\
\hline \multicolumn{5}{|l|}{ Metastasis site } \\
\hline Pleura & $7(33.3)$ & $2(16.7)$ & $5(55.6)$ & 0.159 \\
\hline Contralateral lung & $3(14.3)$ & $2(16.7)$ & $1(11.1)$ & 0.999 \\
\hline Bone & $4(19.0)$ & $3(25.0)$ & $1(11.1)$ & 0.603 \\
\hline Brain & $2(9.5)$ & $1(8.3)$ & $1(11.1)$ & 0.999 \\
\hline Adrenal & $3(14.3)$ & $1(8.3)$ & $2(22.2)$ & 0.553 \\
\hline
\end{tabular}

Values are presented as median (range) or number (\%).

*Statistical comparisons of the data were performed using Mann-Whitney U tests for continuous variables and chi-square tests for categorical variables.

CTC: circulating tumor cell; T: tumor; N: node; M: metastasis.

( $\beta=0.903, p=0.002)$ linear regression analysis (Table 3 ). In the subgroup analysis, the correlation between CTC number and primary tumor volume varied according to the presence of metastasis (Figure 5). CTC number was independent of primary tumor volume $\left(\beta=-0.172, \mathrm{R}^{2}=0.030 ; \mathrm{p}=0.612\right)$ in lung adenocarcinoma patients without distant metastasis. However, CTC number was correlated with primary tumor volume $\left(\beta=0.701, R^{2}=0.491 ; p=0.024\right)$ in lung adenocarcinoma patients with distant metastasis, and more significantly correlated with primary tumor volume $\left(\beta=0.848, \mathrm{R}^{2}=0.719 ; \mathrm{p}=0.033\right)$ in pa- 
Table 2. Baseline characteristics of lung adenocarcinoma patients and healthy controls, before and after propensity matching

\begin{tabular}{|c|c|c|c|}
\hline & Lung adenocarcinoma patients & Healthy controls & p-value \\
\hline \multicolumn{4}{|l|}{ Before matching } \\
\hline Total & 21 & 73 & \\
\hline Male sex & $12(57.1)$ & $45(61.6)$ & $0.710^{*}$ \\
\hline CTC number & $0(0-12)$ & $0(0-2)$ & $<0.001^{*}$ \\
\hline \multicolumn{4}{|l|}{ After matching } \\
\hline Total & 21 & 21 & \\
\hline Age, yr & $65(54-85)$ & $65(53-76)$ & $0.726^{\dagger}$ \\
\hline Male sex & $12(57.1)$ & $12(57.1)$ & $0.999^{+}$ \\
\hline
\end{tabular}

Values are presented as median (range) or number (\%).

*Statistical comparisons of the data were performed using chi-square tests for categorical variables and Mann-Whitney U tests for continu-

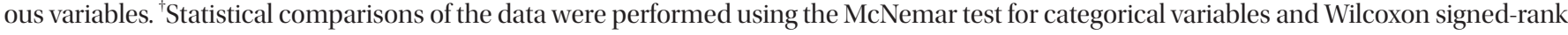
tests for continuous variables.

CTC: circulating tumor cell.

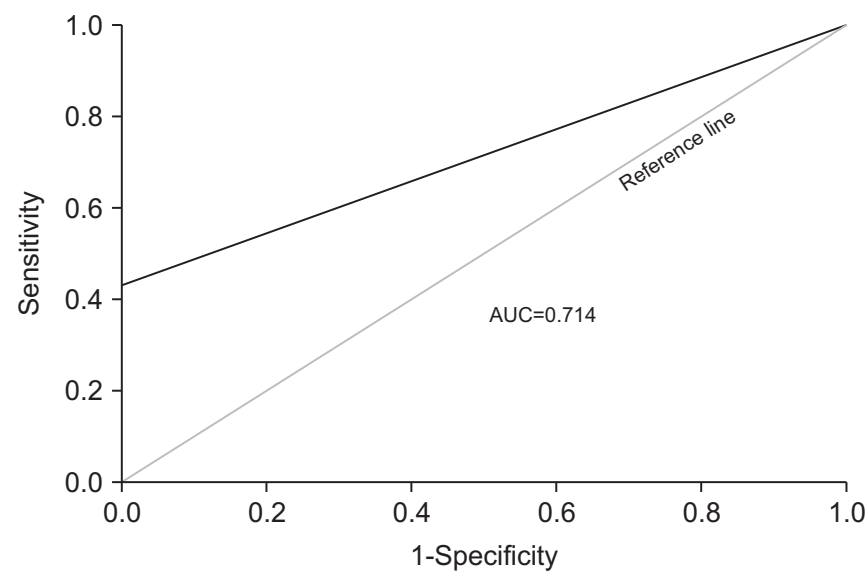

Figure 4. Receiver operating characteristics curve for the diagnostic performance of circulating tumor cell positivity for lung adenocarcinoma, assessed using the propensity score-matched cohort. AUC: area under the curve.

tients with extra-thoracic metastasis. We found an interaction between primary tumor volume (set as a covariable) and the extent of distant metastasis (set as a fixed factor) through the $\operatorname{ANCOVA}(\mathrm{p}=0.036)$.

\section{Discussion}

In this study, we analyzed the relationship between CTC number and primary tumor volume using the FAST-based CTC enrichment method. We hypothesized that the CTC number could be related to tumor volume in lung adenocarcinoma patients. To our knowledge, this is the first study to evaluate the relationship between primary tumor size and CTC number in cancer patients.

FAST is a clog-free and ultrafast centrifugal microfluidic method that allows the enrichment of viable CTCs from whole blood samples of cancer patients, and prior sample treatment is not required. The whole blood sample is injected into the inlet of the loading chamber on the CD-CTC Solo disc cartridge, and CTCs are trapped on a membrane based on their physical characteristics (cell size and rigidity). Kim et al. ${ }^{12}$ reported that the FAST-based CTC enrichment method is highly sensitive, cost-effective, and can be used to detect a significant number of CTCs, not only in cancer patients with distant metastasis, but also in patients in relatively early stages of cancer who do not have distant metastases.

In our analysis performed using the FAST-based CTC enrichment method, we found significant differences in CTC number and AUC results between lung adenocarcinoma patients and healthy individuals. This suggests that CTC number could potentially be a diagnostic marker for lung adenocarcinoma. In addition, the CTC numbers in stage IV lung adenocarcinoma patients were higher than those in stage I-III lung adenocarcinoma patients, suggesting that CTCs could be used as a predictor of distant metastasis and poor prognosis. Our CTC analysis results were in concordance with previous study results ${ }^{9,11,15}$ and suggested that the FAST-based CTC 


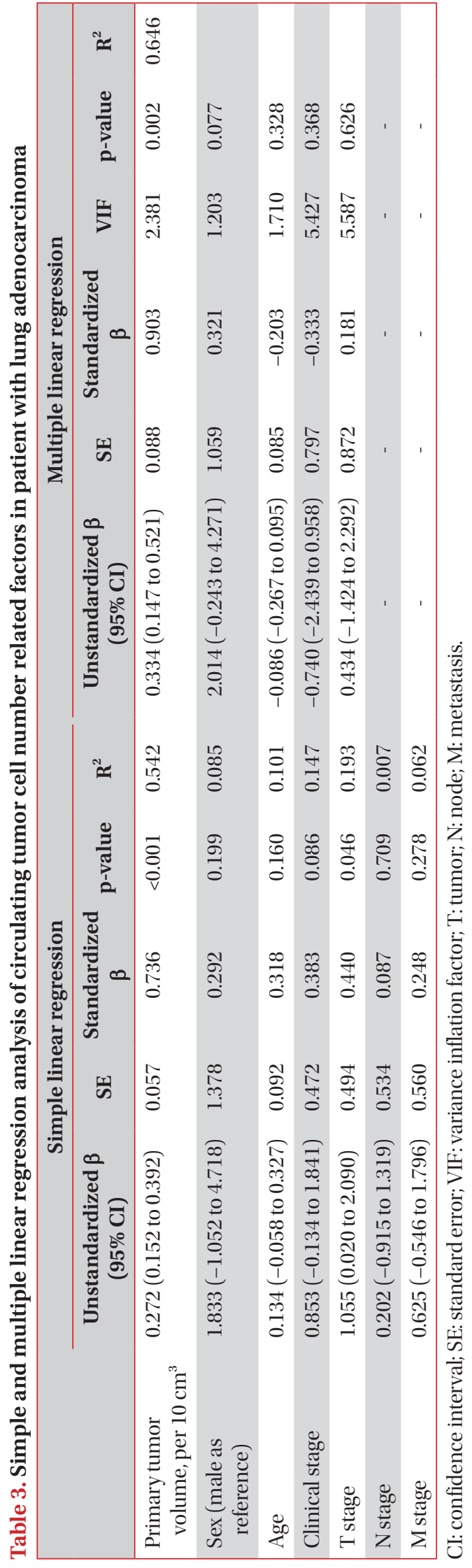

enrichment method might be a useful tool for diagnosis and prognosis in lung adenocarcinoma.

Several studies have attempted to evaluate the associations between clinical factors other than tumor stage and CTC number. Katoh et al. reported a strong correlation between the weight of the primary tumor and CTC number in an animal model $^{16}$. Delfau-Larue et al. ${ }^{17}$ found that tumor metabolic burden, evaluated by positron emission tomography-computed tomography, correlated with the CTC number in follicular lymphoma at diagnosis. In line with these findings, our study also demonstrates that primary tumor volume might be related to CTC number in patients with lung cancer, which may be because tumor volume better reflects cancer burden, like tumor weight and tumor metabolic burden.

Large primary tumor volume and the presence of metastasis are usually observed in advanced-stage cancer. Advancedstage cancer is known to be associated with high CTC numbers $^{9,10}$. We hypothesize that there are more CTCs in cancer patients with large primary tumors and evidence of metastasis. However, an interaction between primary tumor volume and extent of distant metastasis was observed when we analyzed the relationship between CTC number and tumor volume. Our subgroup analysis showed that in patients with metastatic lung adenocarcinoma, there was close relationship between the number of CTCs and primary tumor volume. Fu et al. ${ }^{18}$ found that the number of CTCs did not correlate with whole-body metabolic tumor volume measured by positron emission tomography-computed tomography in IIIB small cell lung cancer patients, and this corresponded with one of our subgroup results (lung adenocarcinoma patients without distant metastasis). We hypothesize that variables in the microenvironment, such as metabolic, stromal, and immunological processes, might prevent cancer metastasis and keep the CTC number low even when the primary tumor volume is large ${ }^{19,20}$. Once the microenvironment is destroyed, cancer cells could spread to other organs and CTC number could increase. A well-designed study is required to draw a definitive conclusion.

Our study had several limitations. First, it was retrospective in nature. Second, it was conducted in a single tertiary referral center, and the sample size was small. Selection bias could not be excluded, and the results should thus be interpreted with caution. In addition, because of the small number of subjects, we could not fully perform a subgroup analysis for factors associated with CTC leakage, and the difference in CTC count according to tumor size, pleural invasion, invasion of great vessels, satellite nodules, and $\mathrm{N}$ or M category (with the same $\mathrm{T}$ category). Thus, further large-scale prospective studies are required. Third, we analyzed CTCs in healthy individuals and patients with lung adenocarcinoma. CTC analysis results from other types of cancer or those obtained using other CTC detection methods might differ from our study results. Fourth, the sample volume used in our study was different 

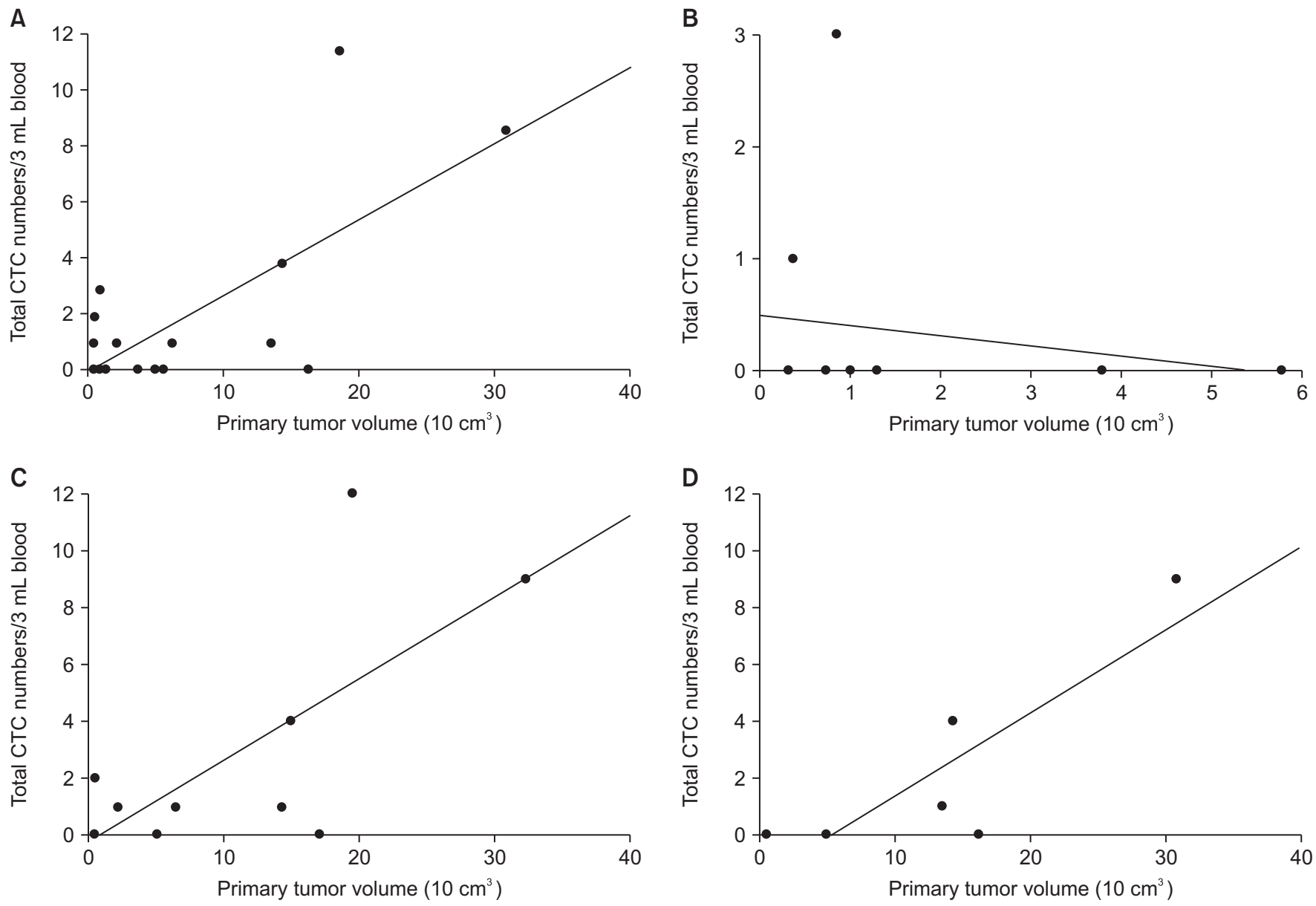

Figure 5. Linear regression model analysis for the association of circulating tumor cell (CTC) number with primary tumor volume. (A) All lung adenocarcinoma patients $(\mathrm{n}=21)\left(\mathrm{R}^{2}=0.542, \beta=0.736, \mathrm{p}<0.001, \mathrm{CTC}=-0.050+0.272 \times\right.$ primary tumor volume $)$. (B) Lung adenocarcinoma patients without distant metastasis $(\mathrm{n}=11)\left(\mathrm{R}^{2}=0.030, \beta=-0.172, \mathrm{p}=0.612\right.$, $\mathrm{CTC}=0.496-0.091 \times$ primary tumor volume $)$. (C) Lung adenocarcinoma patients with distant metastasis $(n=10)\left(R^{2}=0.491, \beta=0.701, p=0.024, C T C=-0.230+0.286 \times\right.$ primary tumor volume). (D) Lung adenocarcinoma patients with extra-thoracic metastasis $(n=6)\left(R^{2}=0.719, \beta=0.848, p=0.033, C T C=-1.558+0.277 \times\right.$ primary tumor volume $)$.

from that of the CellSearch system. Additionally, there were volume differences between samples collected from lung adenocarcinoma patients and healthy individuals, although we adjusted for this discrepancy. Standardization for sample volume or concentration is thus required. Fifth, the FASTbased CTC enrichment method is not an authorized method of CTC analysis. However, there is still no standard method for detecting CTCs in NSCLC patients in clinical practice, and in this study, we presented the clinical utility of FAST-based CTC enrichment method using a propensity-matched healthy individual cohort. Finally, although EpCAM-independent labeling techniques for CTC detection based on the differences in physical properties (size, density, deformability, and electrical properties) have been developed, the sensitivity for CTC detection reported in previous studies and the present one still remain low, and the false positive rate in healthy individuals or those with benign diseases also remains relatively high $^{21-23}$.
Further studies associated with more accurate CTC detection methods are required.

Overall, our study presented the possibility of a correlation between CTC number and primary tumor volume. We showed that CTC numbers might be associated with primary tumor volume in lung adenocarcinomas patients, especially in those with distant metastasis. In addition, CTC analysis by a FAST-based CTC enrichment method might be feasible for detecting and quantifying CTCs in patients with lung adenocarcinoma. Since our study was a retrospective cohort study and included a relatively small number of patients, large-scale prospective studies are required.

\section{Authors' Contributions}

Conceptualization: Ahn JJ, Kim BC. Methodology: Ahn JJ, 
Ra SW. Formal analysis: Ra SW, Kang BJ. Data curation: Lim S. Validation: Lee K, Kim BC. Investigation: Son SH, Kang BJ. Writing - original draft preparation: Kang BJ, Ra SW. Writing - review and editing: Lee K, Kim BC, Lim S, Son SH, Ahn JJ. Approval of final manuscript: all authors.

\section{Conflicts of Interest}

Kyusang Lee, So Hee Son and Byung Chul Kim have conflicts of interest to declare (as members of Clinomics incorporation). Clinomics incorporation financially assisted with the transportation of blood samples and analysis of circulating tumor cells.

\section{Acknowledgments}

We thank the respiratory physicians of Ulsan University Hospital for patient recruitment, and the staff of Clinomics incorporation for assistance in the analysis of circulating tumor cells.

\section{Funding}

No funding to declare.

\section{References}

1. Torre LA, Bray F, Siegel RL, Ferlay J, Lortet-Tieulent J, Jemal A. Global cancer statistics, 2012. CA Cancer J Clin 2015;65:87108.

2. Zhou J, Dong F, Cui F, Xu R, Tang X. The role of circulating tumor cells in evaluation of prognosis and treatment response in advanced non-small-cell lung cancer. Cancer Chemother Pharmacol 2017;79:825-33.

3. Lortet-Tieulent J, Soerjomataram I, Ferlay J, Rutherford M, Weiderpass E, Bray F. International trends in lung cancer incidence by histological subtype: adenocarcinoma stabilizing in men but still increasing in women. Lung Cancer 2014;84:1322.

4. O'Flaherty L, Wikman H, Pantel K. Biology and clinical significance of circulating tumor cell subpopulations in lung cancer. Transl Lung Cancer Res 2017;6:431-43.

5. Yap TA, Lorente D, Omlin A, Olmos D, de Bono JS. Circulating tumor cells: a multifunctional biomarker. Clin Cancer Res 2014;20:2553-68.

6. Han Y, Su C, Liu Z. Methods for detection of circulating cells in non-small cell lung cancer. Front Biosci (Landmark Ed) 2014;19:896-903.

7. Karachaliou N, Mayo-de-Las-Casas C, Molina-Vila MA, Rosell
R. Real-time liquid biopsies become a reality in cancer treatment. Ann Transl Med 2015;3:36.

8. Chen Q, Ge F, Cui W, Wang F, Yang Z, Guo Y, et al. Lung cancer circulating tumor cells isolated by the EpCAMindependent enrichment strategy correlate with cytokeratin 19-derived CYFRA21-1 and pathological staging. Clin Chim Acta 2013;419:57-61.

9. Tanaka F, Yoneda K, Kondo N, Hashimoto M, Takuwa T, Matsumoto $\mathrm{S}$, et al. Circulating tumor cell as a diagnostic marker in primary lung cancer. Clin Cancer Res 2009;15:6980-6.

10. Krebs MG, Sloane R, Priest L, Lancashire L, Hou JM, Greystoke A, et al. Evaluation and prognostic significance of circulating tumor cells in patients with non-small-cell lung cancer. J Clin Oncol 2011;29:1556-63.

11. Lindsay CR, Faugeroux V, Michiels S, Pailler E, Facchinetti F, Ou D, et al. A prospective examination of circulating tumor cell profiles in non-small-cell lung cancer molecular subgroups. Ann Oncol 2017;28:1523-31.

12. Kim TH, Lim M, Park J, Oh JM, Kim H, Jeong H, et al. FAST: size-selective, clog-free isolation of rare cancer cells from whole blood at a liquid-liquid interface. Anal Chem 2017;89:1155-62.

13. Joffe MM, Rosenbaum PR. Invited commentary: propensity scores. Am J Epidemiol 1999;150:327-33.

14. Kim SH, Hong SB, Yun SC, Choi WI, Ahn JJ, Lee YJ, et al. Corticosteroid treatment in critically ill patients with pandemic influenza A/H1N1 2009 infection: analytic strategy using propensity scores. Am J Respir Crit Care Med 2011;183:1207-14.

15. Sonn CH, Cho JH, Kim JW, Kang MS, Lee J, Kim J. Detection of circulating tumor cells in patients with non-small cell lung cancer using a size-based platform. Oncol Lett 2017;13:271722.

16. Katoh M, Neumaier M, Nezam R, Izbicki JR, Schumacher U. Correlation of circulating tumor cells with tumor size and metastatic load in a spontaneous lung metastasis model. Anticancer Res 2004;24:1421-5.

17. Delfau-Larue MH, van der Gucht A, Dupuis J, Jais JP, Nel I, Beldi-Ferchiou A, et al. Total metabolic tumor volume, circulating tumor cells, cell-free DNA: distinct prognostic value in follicular lymphoma. Blood Adv 2018;2:807-16.

18. Fu L, Zhu Y, Jing W, Guo D, Kong L, Yu J. Incorporation of circulating tumor cells and whole-body metabolic tumor volume of (18)F-FDG PET/CT improves prediction of outcome in IIIB stage small-cell lung cancer. Chin J Cancer Res 2018;30:596-604.

19. Lopez-Soto A, Gonzalez S, Smyth MJ, Galluzzi L. Control of metastasis by NK cells. Cancer Cell 2017;32:135-54.

20. Peinado H, Zhang H, Matei IR, Costa-Silva B, Hoshino A, Rodrigues G, et al. Pre-metastatic niches: organ-specific homes for metastases. Nat Rev Cancer 2017;17:302-17.

21. Hosokawa M, Kenmotsu H, Koh Y, Yoshino T, Yoshikawa T, Naito T, et al. Size-based isolation of circulating tumor cells in lung cancer patients using a microcavity array system. PLoS 
One 2013;8:e67466.

22. Chen X, Zhou F, Li X, Yang G, Zhang L, Ren S, et al. Folate receptor-positive circulating tumor cell detected by LT-PCRbased method as a diagnostic biomarker for non-small-cell lung cancer. J Thorac Oncol 2015;10:1163-71.
23. Wang L, Wu C, Qiao L, Yu W, Guo Q, Zhao M, et al. Clinical significance of folate receptor-positive circulating tumor cells detected by ligand-targeted polymerase chain reaction in lung cancer. J Cancer 2017;8:104-10. 